\title{
Comparison of Treatment Outcomes for T3 Glottic Squamous Cell Carcinoma: A Meta-Analysis
}

\author{
Bo Hae Kim¹ · Sung Joon Park ${ }^{2}$ Woo-Jin Jeong ${ }^{3}$ Soon-Hyun Ahn² \\ ${ }^{1}$ Department of Otorhinolaryngology-Head and Neck Surgery, Dongguk University Gyeongju Hospital, Gyeongju; \\ ${ }^{2}$ Department of Otorhinolaryngology-Head and Neck Surgery, Seoul National University Hospital, Seoul; \\ ${ }^{3}$ Department of Otorhinolaryngology-Head and Neck Surgery, Seoul National University Bundang Hospital, Seongnam, Korea
}

\begin{abstract}
Objectives. This study compared the survival outcomes, local control rate, and laryngeal preservation rate of various treatment strategies in the treatment of T3 squamous cell carcinoma of the glottis using proportional meta-analyses.

Methods. Twenty-five retrospective case-series studies were included in these analyses. Treatment strategies were classified as total laryngectomy (TL), open partial laryngectomy (PL), transoral laser microsurgery (TLM), chemo-radiation therapy (CRT), and radiation therapy (RT) alone.

Results. The overall survival rate and disease-specific survival rate among laryngeal preservation treatments did not differ from the overall survival rate of TL. However, the local control rate was lower with RT than TL and PL, and laryngeal preservation rates of TLM and CRT were higher than RT alone.
\end{abstract}

Conclusion. Consideration of preservation of laryngeal function is necessary when treating T3 glottic squamous cell carcinoma. PL, TLM, and, CRT are considered more appropriate initial laryngeal preservation strategies if available.

Keywords. Squamous Cell Carcinoma; Glottis; Survival; Organ Preservation; Meta-Analysis

\section{INTRODUCTION}

T3 glottic cancer was traditionally treated with conventional total laryngectomy (TL) that sacrifices all laryngeal structure. Thus, laryngeal functions loss is an unavoidable morbidity that significantly degrades the quality of life in patients with laryngeal cancer [1-4]. Therefore, treatment modalities including larynx-sparing surgery and nonsurgical treatments to preserve laryngeal function have been introduced [5].

After publication of the landmark Veterans Affairs (VA) and Radiation Therapy Oncology Group (RTOG) 91-11 studies [2,6], there was a major shift in the treatment for advanced laryngeal cancer toward nonsurgical organ preservation strategies [1]. The

\footnotetext{
- Received June 12, 2017

Revised September 6, 2017

Accepted September 8, 2017

- Corresponding author: Soon-Hyun Ahn

Department of Otorhinolaryngology-Head and Neck Surgery,

Seoul National University Hospital, 101 Daehak-ro, Jongno-gu,

Seoul 03080, Korea

Tel: +82-2-2072-7403, Fax: +82-2-2072-4057

E-mail: ahnsh30@snu.ac.kr
}

advances in larynx-sparing surgical technique, including open partial laryngectomy (PL) and transoral laser microsurgery (TLM), have broadened the treatment of T3 glottic cancers [7-10]. Interestingly, laryngeal cancer is one of the few cancers that have decreased in survival over the past 30 years [1], and some authors suggest that this result may result from nonsurgical organ preservation treatment in advanced laryngeal cancer [1,11]. Because T3 squamous cell carcinoma ( $\mathrm{SqCC}$ ) of the glottis is a heterogenous group defined as in tumor with vocal fold fixation, considerable invasion of the paraglottic structure, or inner cortex invasion of the thyroid cartilage [8], various primary treatments are being used in accordance with physician's decision. Therefore, while primary treatment modalities show good oncological outcome, only a few comparative studies have been reported T3 specific oncological results of treatments $[8,12]$, and the choice of primary treatment is unsettled, especially in the glottis, because of inconsistent treatment results.

For this reason, this study compares the primary survival outcome, local control (LC) rate, and laryngeal preservation (LP) rate of treatment strategies of $3 \mathrm{SqCC}$ of the glottis.

Copyright @ 2018 by Korean Society of Otorhinolaryngology-Head and Neck Surgery.

This is an open-access article distributed under the terms of the Creative Commons Attribution Non-Commercial License (http://creativecommons.org/licenses/by-nc/4.0)

which permits unrestricted non-commercial use, distribution, and reproduction in any medium, provided the original work is properly cited. 


\section{MATERIALS AND METHODS}

\section{Search strategy and study selection}

The purpose of this study was to compare the outcomes of primary treatment modalities for $\mathrm{T} 3 \mathrm{SqCC}$ of the glottis. English literature searching in the databases of US National Library of Medicine (MEDLINE) and Excerpta Medica database (EMBASE) was conducted to identify all studies concerning treatments in T3 SqCC of the glottis from January 1965 to November 2016. The last search was done on November 4, 2016 and the following search strategy was used: advanced/extended/T3, glottis/vocal, and carcinoma/cancer/squamous.

Studies were included if they fulfilled the following criteria: (1) published literature in English language (2) for the treatment in patients with untreated and curable stage (3) including at least one of 5-year outcomes among LC rate, LP rate, overall survival (OS) rate, or disease-specific survival (DSS) rate after the treatment of T3 SqCC in the glottis, and (4) treatment modalities: TLM,TL, PL, RT (radiation therapy; any protocol of curative RT, 60-75 Gy on primary site), or CRT (chemo-radiation therapy; RT with platinum-based regimen).

The titles and abstracts identified by the literature search were independently screened by two reviewers (BHK, SJP). All published studies of the treatment of SqCC of the glottis were considered if they evaluated at least 10 consecutive patients. This limitation was made to exclude small case series study or case reports. Full review of publications after abstract review was performed to assess eligibility, and data were extracted by two reviewers. The adjustment of T stage according to American Joint Committee on Cancer seventh edition was conducted in this step if necessary [13]. In addition, where there were multiple publications using an overlapping patient pool, large studies were included, but published abstracts without full manuscript, comments, editorials, and letters to editor were excluded.

\section{HIIGHLIIGIHTS}

- Twenty-five studies were included for analyses of outcomes of T3 glottic squamous cell carcinoma (SqCC).

- The overall survival rate of transoral laser microsurgery (TLM), chemo-radiation therapy (CRT), and radiation alone (RT) in patients with $\mathrm{T} 3 \mathrm{SqCC}$ of the glottis did not differ from the overall survival rate of total laryngectomy (TL).

- There were no significant differences in the disease-specific survival rate by TL, TLM, or RT.

- Local control (LC) rates were similar with TL, open partial laryngectomy (PL), and CRT although the LC rate of RT was poorer than TL and PL.

- The laryngeal preservation rate of TLM and CRT was higher than with RT.

\section{Data collection and quality assessment}

The name of the first author, year of publication, study design, number of included patients, and primary treatment modalities were collected from the included studies. Survival outcomes including OS and DSS rate were collected from the studies, and LC and LP rate were additionally collected or calculated. In patients who underwent TLM, re-resection rate was calculated from included studies if available. Data extraction was conducted by two reviewers using a uniform sheet for collecting data. Any disagreements of extracted data were resolved through discussion between the two reviewers to reach a consensus or by consulting a third reviewer if necessary. In addition, although there is no formally accepted quality assessment tool for case series, National Institute for Health and Care Excellence (NICE) tool for quality assessment of case-series [14], composed of eight questions and represented with a score (Supplementary Table 1), was used to assist understanding of a study by the two reviewers.

\section{Statistical analysis}

Proportional meta-analyses were performed to evaluate the rate of OS, DSS, LC, and LP by treatment using a random-effects model because of the uncontrolled variables in the included studies [15]. The results of meta-analyses were presented with a forest plot, and the length of horizontal lines in forest plot corresponded to $95 \%$ confidential interval (CI) of effect estimate of each study. When we compared outcomes of primary treatment modalities, overlap of $95 \% \mathrm{CI}$ between treatments outcomes was defined as failure to reject the null hypothesis of no difference among treatments [15]. The Cochran $\mathrm{Q}$ and $\mathrm{I}^{2}$ test was used to assess heterogeneity of the included studies. Cochran Q $P<0.10$ or $I^{2}>50 \%$ was defined as heterogeneity of the included studies [16]. $\mathrm{I}^{2}$ is the proportion of total variation detected between included studies. A relationship between effect size and precision to evaluate the possibility of publication bias was determined by Egger's regression test [17]. Statistical analyses were carried out using StatsDirect software ver. 3.0.184 (StatsDirect, Cheshire, UK), and these results were reportrayed as forest plot by an excel program using described methods [18].

\section{RESULTS}

\section{Search results and quality of included studies}

The literature search yielded 1,228 abstracts identified from the MEDLINE and EMBASE. Randomized clinical trials, case-control studies, and prospective studies were not identified in the literature search. Therefore, meta-analyses were conducted using case-series studies. We excluded 1,164 studies which did not meet the inclusion criteria through abstract review, and 64 articles were fully reviewed for data collection (Fig. 1). Among the 64 fully reviewed studies, 39 studies were additionally excluded 
due to lack of relevant data for analysis in 30 studies, registry or survey data in two studies, and duplicated clinical data in seven studies. Therefore, only 25 retrospective case-series studies were eligible for meta-analyses by our inclusion criteria (Table 1) $[8,10,19-41]$. Three studies included treatment outcomes of

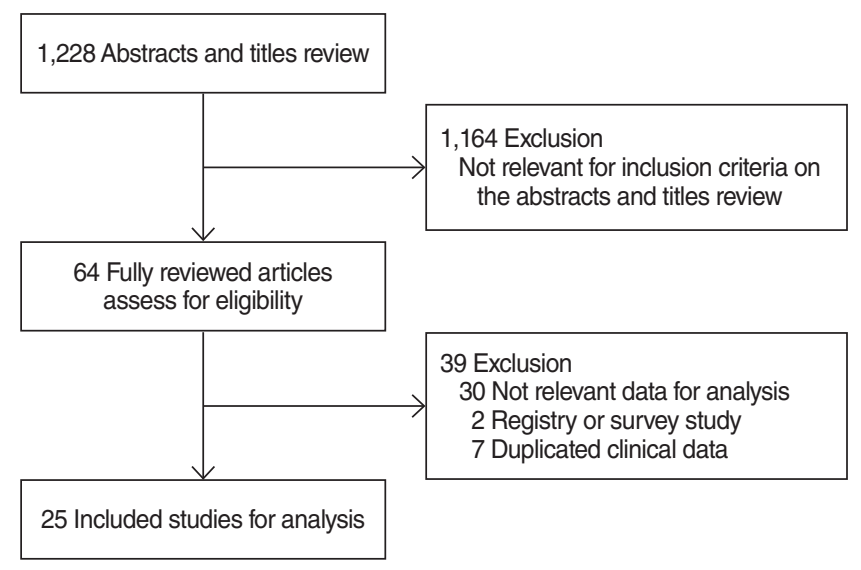

Fig. 1. Flow chart of studies through the review. more than two different treatments.

The primary treatment of T3 SqCC of the glottis was TL in 352 patients (six studies), PL in 66 patients (two studies), TLM in 374 patients (nine studies), RT in 452 patients (eight studies), and CRT in 73 patients (three studies). Although meta-analyses were conducted only in relation to the primary treatment, and subsequent (secondary) analysis between primary treatments with adjuvant therapy and without adjuvant therapy was difficult because of small sample size. As a result, 1,317 patients diagnosed as glotticT3 SqCC were included in this analysis, and the number of enrolled patients in each study varied from 11 to 176 . The quality assessment score for included studies on the NICE tool varied from 4 to 6 (Table 1), but all studies regardless of quality score were included for meta-analyses to maximize sample size.

\section{Overall survival rate}

The results of OS were reported in 858 patients of 16 studies; OS rate was highest for 46 patients who underwent CRT (0.870; $95 \% \mathrm{CI}, 0.622$ to $0.994 ; \mathrm{I}^{2}=74.8 \%$ ). However, there were not significant differences in the OS rate compared with TL $(0.557$; $95 \%$ CI, 0.501 to $0.633 ; I^{2}=0 \%$ ), In addition, OS rate of TLM

Table 1. A brief summary of included studies

\begin{tabular}{|c|c|c|c|c|c|c|c|c|c|c|c|c|c|c|}
\hline \multirow{2}{*}{ Treatment } & \multirow{2}{*}{ First author } & \multirow{2}{*}{ Year } & \multirow{2}{*}{ Country } & \multirow{2}{*}{$\begin{array}{c}\text { No. of } \\
\text { patients } \\
(n=1,317)\end{array}$} & \multirow{2}{*}{$\begin{array}{l}\text { Available } \\
\text { outcome }\end{array}$} & \multicolumn{9}{|c|}{ Quality assessment } \\
\hline & & & & & & Q1 & Q2 & Q3 & Q4 & Q5 & Q6 & Q7 & Q8 & Total \\
\hline \multirow[t]{6}{*}{ TL } & Porter et al. [19] & 1998 & New Zealand & 19 & LC, OS, DSS & 0 & 1 & 1 & 1 & 0 & 0 & 1 & 1 & 5 \\
\hline & Kowalski et al. [20] & 1996 & Brazil & 176 & LC, OS & 0 & 1 & 1 & 1 & 0 & 1 & 0 & 1 & 5 \\
\hline & Foote et al. [21] & 1994 & US & 81 & LC, OS, DSS & 0 & 1 & 1 & 1 & 0 & 0 & 1 & 1 & 5 \\
\hline & Simpson et al. [22] & $1993^{\text {a) }}$ & Scotland & 36 & OS & 0 & 1 & 1 & 1 & 0 & 0 & 1 & 1 & 5 \\
\hline & Woodhouse et al. [23] & 1981 & US & 16 & LC & 0 & 1 & 1 & 1 & 0 & 0 & 1 & 1 & 5 \\
\hline & Harwood et al. [24] & $1979^{b)}$ & Canada & 24 & LC & 0 & 1 & 1 & 1 & 0 & 0 & 1 & 1 & 5 \\
\hline \multirow[t]{2}{*}{$\mathrm{PL}$} & Nakayama et al. [25] & 2015 & Japan & 26 & LC & 0 & 1 & 1 & 1 & 0 & 1 & 0 & 1 & 5 \\
\hline & Leszczynska et al. [26] & 2015 & Poland & 40 & LC, LP & 0 & 1 & 1 & 1 & 0 & 0 & 1 & 1 & 5 \\
\hline \multirow[t]{9}{*}{ TLM } & Day et al. [27] & 2017 & US & 12 & LC, LP, OS, DSS & 0 & 1 & 1 & 1 & 1 & 0 & 1 & 1 & 6 \\
\hline & Ansarin et al. [28] & 2017 & Italy & 36 & LP, DSS & 0 & 1 & 1 & 1 & 0 & 0 & 1 & 1 & 5 \\
\hline & Peretti et al. [29] & 2016 & Italy & 34 & LC, LP, OS & 0 & 1 & 1 & 1 & 0 & 0 & 1 & 1 & 5 \\
\hline & Breda et al. [30] & 2015 & Portugal & 26 & LP, DSS & 0 & 1 & 0 & 1 & 0 & 1 & 1 & 1 & 5 \\
\hline & Pantazis et al. [31] & 2015 & Greece & 19 & LC, LP, OS, DSS & 0 & 1 & 1 & 1 & 0 & 1 & 1 & 1 & 6 \\
\hline & Canis et al. [10] & 2014 & Germany & 122 & LC, OS, DSS & 0 & 1 & 1 & 1 & 0 & 0 & 1 & 1 & 5 \\
\hline & Mantsopoulos et al. [8] & 2012 & Germany & 23 & LP & 0 & 1 & 1 & 1 & 0 & 1 & 1 & 1 & 6 \\
\hline & Vilaseca et al. [32] & 2010 & Spain & 51 & LC, LP, OS, DSS & 0 & 1 & 1 & 1 & 0 & 0 & 1 & 1 & 5 \\
\hline & Motta et al. [33] & 2005 & Italy & 51 & LC, OS, DSS & 0 & 1 & 1 & 1 & 0 & 0 & 1 & 1 & 5 \\
\hline \multirow[t]{8}{*}{ RT } & Hinerman et al. [34] & 2007 & US & 87 & LC, OS, DSS & 0 & 1 & 1 & 1 & 0 & 0 & 1 & 1 & 5 \\
\hline & Murakami et al. [35] & $2006^{c}$ & Japan & 22 & $\mathrm{LC}, \mathrm{LP}$ & 0 & 1 & 1 & 1 & 0 & 0 & 1 & 1 & 5 \\
\hline & Jorgensen et al. [36] & 2002 & Denmark & 81 & DSS & 0 & 1 & 1 & 1 & 0 & 1 & 1 & 1 & 6 \\
\hline & MacKenzie et al. [37] & 2000 & Canada & 11 & OS, DSS & 0 & 1 & 1 & 1 & 0 & 0 & 1 & 1 & 5 \\
\hline & Mendenhall et al. [38] & 1997 & US & 75 & LC, OS, DSS & 0 & 1 & 1 & 1 & 0 & 0 & 1 & 1 & 5 \\
\hline & Simpson et al. [22] & $1993^{\text {a) }}$ & Scotland & 38 & OS & 0 & 1 & 1 & 1 & 0 & 0 & 1 & 1 & 5 \\
\hline & Wang et al. [39] & 1986 & US & 70 & LC & 0 & 1 & 1 & 1 & 0 & 1 & 1 & 1 & 6 \\
\hline & Harwood et al. [24] & $1979^{b)}$ & Canada & 68 & LC, LP, DSS & 0 & 1 & 1 & 1 & 0 & 0 & 1 & 1 & 5 \\
\hline \multirow[t]{3}{*}{ CRT } & Furusaka et al. [40] & 2013 & Japan & 29 & LP, OS & 0 & 1 & 1 & 1 & 0 & 0 & 1 & 0 & 4 \\
\hline & Lin et al. [41] & 2012 & Australia & 17 & LC, LP, OS & 0 & 1 & 1 & 1 & 0 & 0 & 1 & 1 & 5 \\
\hline & Murakami et al. [35] & $2006^{c)}$ & Japan & 27 & LC, LP & 0 & 1 & 1 & 1 & 0 & 0 & 1 & 1 & 5 \\
\hline
\end{tabular}

TL, total laryngectomy; LC, local control; OS, overall survival; DSS, disease-specific survival; PL, open partial laryngectomy; LP, laryngeal preservation; TLM, transoral laser microsurgery; RT, radiation therapy; CRT, chemo-radiation therapy.

a-c) Studies including outcomes from different treatment modalities. 


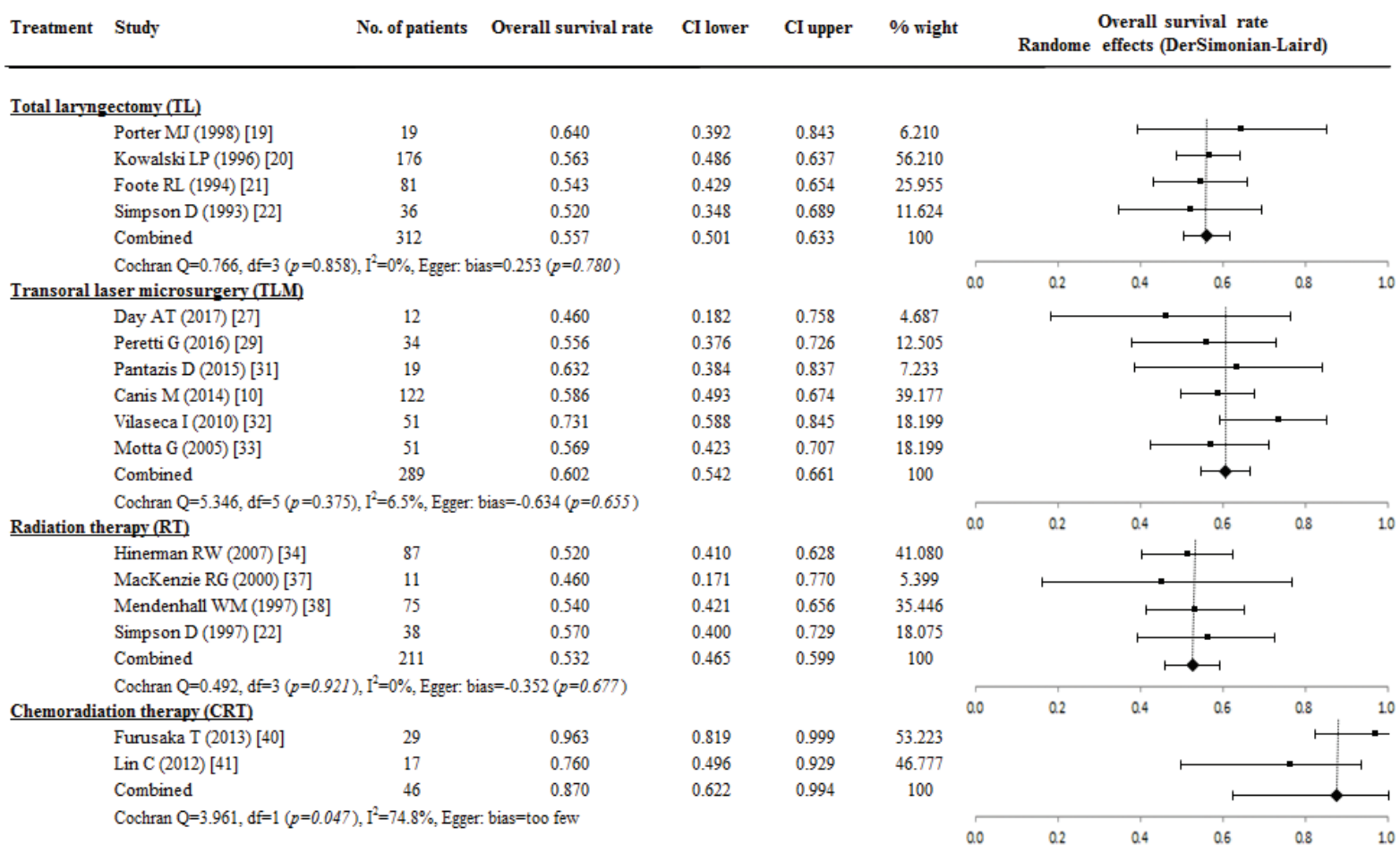

Fig. 2. A proportional meta-analysis of case series studies comparing overall survival rate after primary treatments in patients with T3 glottic squamous cell carcinoma.

(0.602; $95 \%$ CI, 0.542 to $\left.0.661 ; I^{2}=6.5 \%\right)$ and RT $(0.532 ; 95 \%$ CI, 0.465 to $0.599 ; \mathrm{I}^{2}=0 \%$ ) did not differ significantly from the OS rate of TL. The possibility of publication bias on OS rate presented in CRT $(P=0.047)$ (Fig. 2).

\section{Disease-specific survival rate}

Fourteen studies of TL (two studies, 100 patients), TLM (seven studies, 317 patients), and RT (five studies, 322 patients) group were available for the analysis of the DSS rate. There were no significant differences of DSS rate among TL $(0.719 ; 95 \% \mathrm{CI}$, 0.628 to $\left.0.802, I^{2}=0 \%\right)$, TLM $(0.807 ; 95 \%$ CI, 0.735 to 0.870 ; $\left.\mathrm{I}^{2}=52.3 \%\right)$ and RT $\left(0.723 ; 95 \% \mathrm{CI}, 0.622\right.$ to $\left.0.813 ; \mathrm{I}^{2}=71.2 \%\right)$ group. The $P$-value of the Egger's test was 0.125 in TLM and 0.563 in RT group (Fig. 3).

\section{Local control rate}

The comparison of $\mathrm{LC}$ rate was available for meta-analysis in five studies of TL (316 patients), two studies of PL (66 patients), six studies of TLM (289 patients), five studies in RT (322 patients), and two studies of CRT (41 patients). Adjuvant therapy after primary treatment in patients with not enough resection margin or remnant tumors was provided to patients in all studies, but the exact rate of adjuvant therapy after treatment was difficult to calculate because of inconsistent adjuvant therapy description. The evaluation of the re-resection rate in patients who underwent TLM was available in three studies, and $16.7 \%$ (28/168 patients) underwent re-resection for proper resection margin.

The highest pooled proportion of LC rate estimated from a random effect model was 0.839 (95\% CI, 0.742 to 0.917 ; $\left.\mathrm{I}^{2}=0 \%\right)$ in 66 patients who received PL, but this results did not significantly differ from 0.773 of TL group $(95 \%$ CI, 0.647 to $0.877 ; \mathrm{I}^{2}=77.8 \%$ ), 0.643 of TLM ( $95 \%$ CI, 0.537 to 0.742 ; $\mathrm{I}^{2}=65.8 \%$ ), and 0.743 of CRT group (95\% CI, 0.471 to 0.939 ; $\left.\mathrm{I}^{2}=70.5 \%\right)$. However, LC rate after RT was 0.504 (95\% CI, 0.373 to $0.634 ; \mathrm{I}^{2}=82.1 \%$ ), and this result was a poor $\mathrm{LC}$ rate compared with TL and PL group. The effect of study size was not significantly related with LC rate in TLM $(P=0.408)$, TL $(P=0.408)$, and RT $(P=0.348)$ group when we evaluated the possibility of publication bias using Egger's (Fig. 4).

\section{Laryngeal preservation rate}

The LP rate was available in 361 patients from the TLM treatment (seven studies, 201 patients), RT (two studies, 90 patients), and CRT (three studies, 70 patients) group. The overall proportional of LP rate in patients receiving TLM was 0.761 (95\% CI, 0.635 to $0.868 ; I^{2}=73.9 \%$ ), and TLM was not significantly associated with LP rate and study size $(P=0.358)$. The LP rate did not differ between TLM and CRT $(0.842 ; 95 \%$ CI, 0.738 to 0.924; $\left.\mathrm{I}^{2}=18.2 \%\right)$. However, the LP rate of TLM and CRT was higher than with RT $\left(0.439 ; 95 \%\right.$ CI, 0.339 to $\left.0.542 ; \mathrm{I}^{2}=0 \%\right)$ (Fig. 5). 


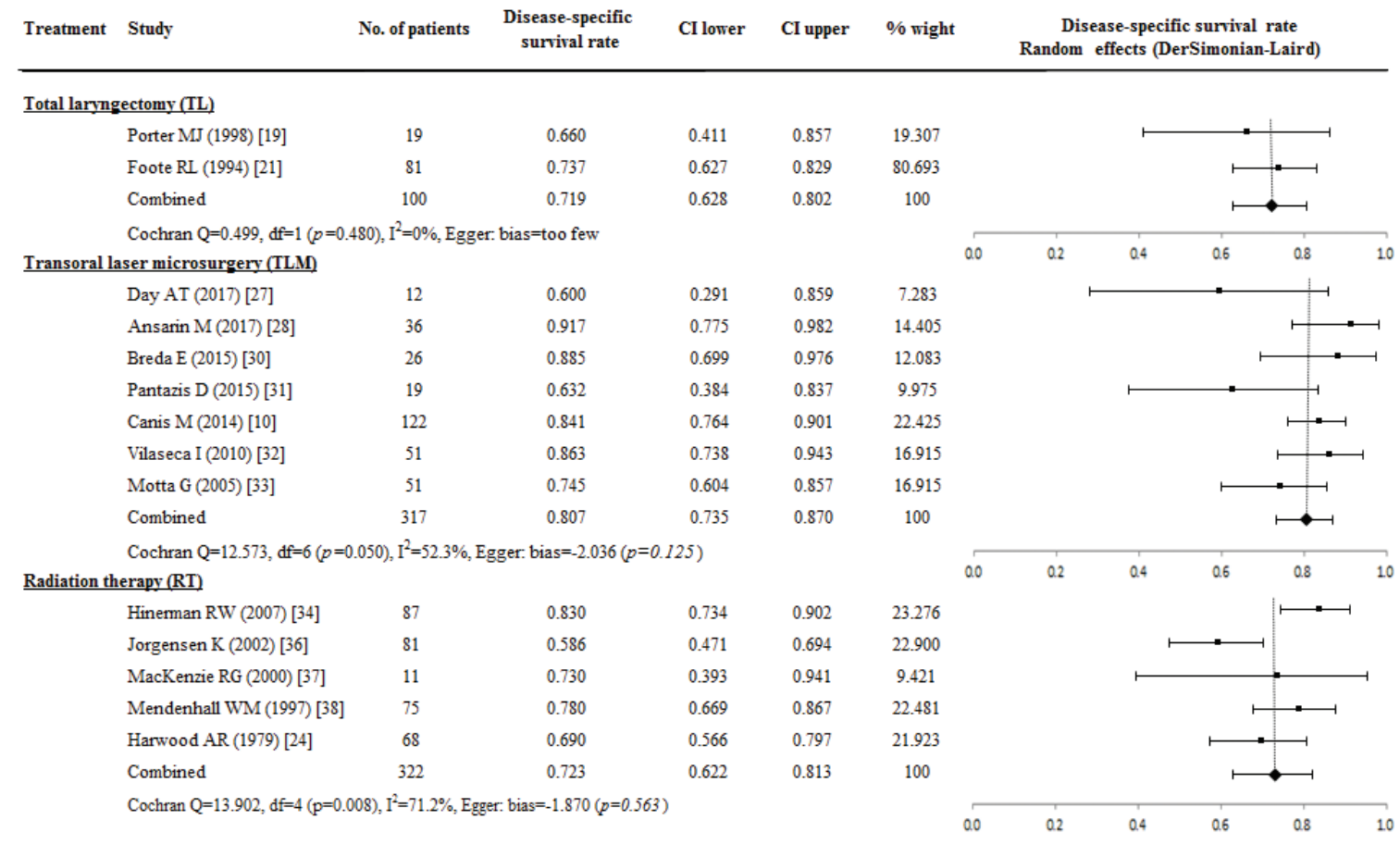

Fig. 3. A proportional meta-analysis of case series studies comparing disease-specific survival rate after primary treatments in patients with T3 glottic squamous cell carcinoma.

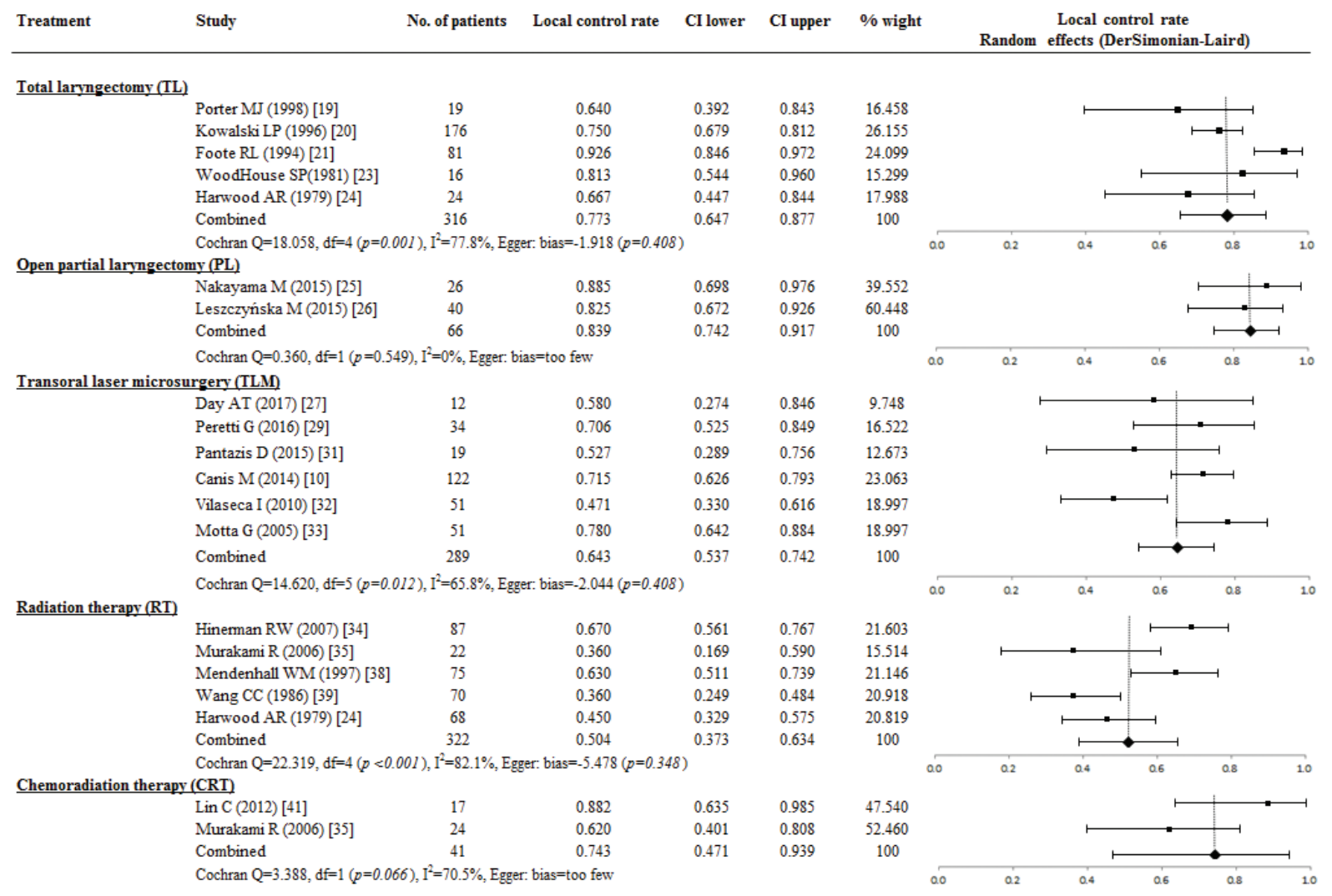

Fig. 4. A proportional meta-analysis of case series studies comparing local control rate after primary treatments in patients with T3 glottic squamous cell carcinoma. 


\begin{tabular}{|c|c|c|c|}
\hline Treatment & Study & No. of patients & Laryngeal preservati \\
\hline \multicolumn{4}{|c|}{ Transoral laser microsurgery (TLM) } \\
\hline & Day AT (2017) [27] & 12 & 0.806 \\
\hline & Ansarin M (2017) [28] & 36 & 0.806 \\
\hline & Peretti G (2016) [29] & 34 & 0.853 \\
\hline & Breda E (2015) [30] & 26 & 0.692 \\
\hline & Pantazis D (2015) [31] & 19 & 0.737 \\
\hline & Mantsopoulos K (2012) [8] & 23 & 0.956 \\
\hline & Vilaseca I (2010) [32] & 51 & 0.510 \\
\hline & Combined & 201 & 0.761 \\
\hline & \multicolumn{3}{|c|}{ Cochran $\mathrm{Q}=23.030, \mathrm{df}=6(p<0.001), \mathrm{I}^{2}=73.9 \%$, Egger: bias $=-3.201(p=0.358)$} \\
\hline \multicolumn{4}{|c|}{ Radiation therapy (RT) } \\
\hline & Murakami R (2006) [35] & 22 & 0.400 \\
\hline & Harwood AR (1979) [24] & 68 & 0.450 \\
\hline & Combined & 90 & 0.439 \\
\hline & \multicolumn{3}{|c|}{ Cochran $Q=0.150, \mathrm{df}=1(p=0.699), \mathrm{I}^{2}=0 \%$, Egger: bias=too few } \\
\hline \multicolumn{4}{|c|}{ Chemoradiation therapy (CRT) } \\
\hline & Furusaka T (2013) [40] & 29 & 0.925 \\
\hline & $\operatorname{Lin} C(2012)[41]$ & 17 & 0.760 \\
\hline & Murakami R (2006) [35] & 24 & 0.830 \\
\hline & Combined & 70 & 0.842 \\
\hline & \multicolumn{3}{|c|}{ Cochran $\mathrm{Q}=2.446, \mathrm{df}=2(p=0.294), \mathrm{I}^{2}=18.2 \%$, Egger: bias $=$ too few } \\
\hline
\end{tabular}
CI lower CI upper $\%$ wight $\quad \begin{gathered}\text { Laryngeal preservation rate } \\ \text { Random effects (DerSimonian-Laird) }\end{gathered}$

Fig. 5. A proportional meta-analysis of case series studies comparing laryngeal preservation rate after primary treatments in patients with T3 glottic squamous cell carcinoma.

\section{DISCUSSION}

TL was traditionally considered the primary option to treat $\mathrm{T} 3$ laryngeal cancer until radiotherapy or chemo-radiotherapy became popular treatment [12]. As the nonsurgical treatments could preserve the larynx and provide acceptable treatment for laryngeal cancers after publication of the VA and RTOG 91-11 studies [2,6], CRT use has become widespread for primary treatment of advanced laryngeal cancers [42,43]. In addition, over that periods, the surgical techniques that preserve laryngeal functions have developed and improved, and open PL, which spares at least one cricoarytenoid unit, and TLM, that expands its indication for advanced laryngeal cancer, have evolved to primary treatment for selected advanced laryngeal cancers $[9,10,44]$. Therefore, several treatment options are available as the primary treatment in T3 glottic cancer. Understanding the efficacy of each modality is important for providing appropriate treatment.

Although TL treatment of advanced laryngeal cancer has decreased over time, TL is still considered the most effective option for primary treatment of advanced laryngeal cancer [12]. Therefore, the VA trial probed the efficacy of nonsurgical strategies in preserving the larynx compared with TL [2]. In this study, the survival rate was compared with that of TL to analyze the effectiveness of each treatment modality. Fu et al. [5] reported a comparative meta-analysis of T3 specific survival rate including OS and DSS in advanced laryngeal cancers, and observed no significant difference of survival between TL and nonsurgical treatment. We likewise found no significant differences of OS rate of all type of laryngeal preservation strategies including TLM (0.602; 95\% CI, 0.542 to $\left.0.661 ; \mathrm{I}^{2}=6.5 \%\right)$, RT $(0.532 ; 95 \% \mathrm{CI}$, 0.465 to $\left.0.599 ; \mathrm{I}^{2}=0 \%\right)$, and CRT compared to TL $(0.557 ; 95 \%$ CI, 0.501 to $\left.0.633 ; I^{2}=0 \%\right)$. Surgical treatments are described as yielding better OS outcome compared with nonsurgical treatments in advanced laryngeal cancer treatment [45]. In contrast, in our study, CRT had the highest OS rate. In contrast to the RTOG 91-11 study, which reported higher loco-regional control rate, not a survival benefit of CRT in advanced laryngeal cancers [6], our results yielded a significantly higher OS rate compared to RT alone. Some authors have argued that decreased survival outcome in patients with advanced laryngeal cancer may be induced by laryngeal preservation strategies [1], especially nonsurgical treatment. Our result suggests that TLM, RT, and CRT are feasible primary options for the treatment of T3 glottic SqCC when we consider sustainability of laryngeal function, and the advantage of TL in the treatment of T3 glottic SqCC is not obvious. In addition, when we use nonsurgical treatment for primary treatment, CRT is better initial option from the perspective of OS rate compared to RT alone. However, there were no significant differences of DSS rate among TL, TLM, and RT.

In this analysis, $\mathrm{PL}$ gave the highest $\mathrm{LC}$ rate $(0.839 ; 95 \% \mathrm{CI}$, 0.742 to $0.917 ; \mathrm{I}^{2}=0 \%$ ). Because it is important to ensure that adequate margins are obtained when excising a primary tumor of the larynx, PL, which preserves at least one cricoarytenoid unit, was used in selected cases [7,9]. LC rate and survival outcome after open PL were similar with TL in selected cases $[25,46]$. Consistent with this result, even though only LC rate 
could be analyzed, LC rate of PL did not differ significantly from the LC rate of TL. Additionally, the critical point of TLM use in the treatment of glottis cancer is the exposure of the entire site where the tumor invaded for proper resection [31]. Although $16.7 \%$ of re-resection was needed for proper resection margin in TLM group, there is no significant difference with that of TL $(0.643 ; 95 \%$ CI, 0.537 to 0.742 vs. $0.773 ; 95 \%$ CI, 0.647 to $0.877 ; \mathrm{I}^{2}=77.8 \%$ ) in aspect of $\mathrm{LC}$ rate. Although this analysis was conducted without detailed classification according to invasion site, the LP rate of TLM did not differ significantly from the CRT. Thus, although definite indication or limitation is not established, TLM may be an option for primary treatment in selective T3 cases. Therefore, we concluded that PL and TLM are available options for initial surgical treatment in T3 glottic SqCC from the perspective of LC and LP.

Despite good results of surgical modalities, nonsurgical treatments for laryngeal cancer are widely used as primary treatment because of good oncological and LP rate. However, RT alone showed significantly poor LC rate compared with TL, PL, TLM and CRT $\left(0.743 ; 95 \%\right.$ CI, 0.471 to $\left.0.939 ; \mathrm{I}^{2}=70.5 \%\right)$. In contrast to RT alone, CRT LC rate is comparable with TL, consistent results with the RTOG 91-11 study [6]. This result agrees with studies that reported better outcome of CRT than RT alone in advanced laryngeal cancers, favoring CRT [6,34]. Although inconsistent oncological results of RT alone have been noted, LP after the RT alone treatment was worse than TLM and CRT.

The limitations of these meta-analyses are presented. First, almost all survival data of TL included this analysis were described in the 1990s. Therefore, this data might not reflect the current state of TL results. Second, TLM yields good oncological results in several studies. However, the TLM procedures included in this analysis were performed only by experts. Therefore, we conclude that TLM lacks evidence for its popular use in T3 glottic cancers. Third, most studies of the efficacy of nonsurgical strategies for laryngeal cancer cannot be included because T3 and T4 patients are mixed in many cases without separated description of survival or LP according to T stage, or survival outcome was not classified by location of the tumor between glottis and supra-glottis. Fourth, the design of this study was to compare T3specific oncological outcomes according to treatment modalities. Therefore, the comparison of functional outcomes, such as voice and swallowing, was not able to be demonstrated according to treatment modalities. Also, there were not enough descriptions regarding functional results based on treatment modalities in most of the studies included in our meta-analysis. Further study should be conducted focused on comparing the functional results of each treatment modalities.

In conclusion, all of the laryngeal preservation strategies, including surgical and nonsurgical treatment, have similar survival outcomes compared to TL. Therefore, laryngeal preservation strategies including surgical and nonsurgical treatment are reasonable options for T3 glottic SqCC from the perspective of sur- vival outcome. Thus, consideration of sustainability of laryngeal function is necessary in T3 glottic cancer, and laryngeal preservation strategies including PL, TLM, and CRT are considered more appropriate initial strategies if available. However, RT alone has a limited LC and LP rate compared to other laryngeal preservation strategies. Therefore, RT alone is considered not an adequate option for primary treatment.

\section{CONFLICT OF INTEREST}

No potential conflict of interest relevant to this article was reported.

\section{SUPPLEMENTARY MATERIAL}

Supplementary Table 1. National Institute for Health and Care Excellence (NICE) tool for quality assessment of case-series. It can be found via https://doi.org/10.21053/ceo.2017.00717.

\section{REFERENCES}

1. Hoffman HT, Porter K, Karnell LH, Cooper JS, Weber RS, Langer CJ, et al. Laryngeal cancer in the United States: changes in demographics, patterns of care, and survival. Laryngoscope. 2006 Sep;116 (9 Pt 2 Suppl 111):1-13.

2. Department of Veterans Affairs Laryngeal Cancer Study Group,Wolf GT, Fisher SG, Hong WK, Hillman R, Spaulding M, et al. Induction chemotherapy plus radiation compared with surgery plus radiation in patients with advanced laryngeal cancer. N Engl J Med. 1991 Jun;324(24):1685-90.

3. Forastiere AA, Zhang Q, Weber RS, Maor MH, Goepfert H, PajakTF, et al. Long-term results of RTOG 91-11: a comparison of three nonsurgical treatment strategies to preserve the larynx in patients with locally advanced larynx cancer. J Clin Oncol. 2013 Mar;31(7):845-52.

4. McNeil BJ, Weichselbaum R, Pauker SG. Speech and survival: tradeoffs between quality and quantity of life in laryngeal cancer. $\mathrm{N}$ Engl J Med. 1981 Oct;305(17):982-7.

5. Fu X, Zhou Q, Zhang X. Efficacy comparison between total laryngectomy and nonsurgical organ-preservation modalities in treatment of advanced stage laryngeal cancer: a meta-analysis. Medicine (Baltimore). 2016 Apr;95(14):e3142.

6. Forastiere AA, Goepfert H, Maor M, PajakTF, Weber R, Morrison W, et al. Concurrent chemotherapy and radiotherapy for organ preservation in advanced laryngeal cancer. N Engl J Med. 2003 Nov;349 (22):2091-8.

7. Korean Society of Thyroid-Head and Neck Surgery Guideline Task Force, Ahn SH, Hong HJ, Kwon SY, Kwon KH, Roh JL, et al. Guidelines for the Surgical Management of Laryngeal Cancer: Korean Society of Thyroid-Head and Neck Surgery. Clin Exp Otorhinolaryngol. 2017 Mar;10(1):1-43.

8. Mantsopoulos K, Psychogios G, Bohr C, Zenk J, Kapsreiter M,Waldfahrer F, et al. Primary surgical treatment of T3 glottic carcinoma: long-term results and decision-making aspects. Laryngoscope. 2012 Dec;122(12):2723-7.

9. American Society of Clinical Oncology, Pfister DG, Laurie SA, Weinstein GS, Mendenhall WM, Adelstein DJ, et al. American Society of Clinical Oncology clinical practice guideline for the use of larynx- 
preservation strategies in the treatment of laryngeal cancer. J Clin Oncol. 2006 Aug;24(22):3693-704.

10. Canis M, Ihler F, Martin A, Wolff HA, Matthias C, SteinerW. Results of 226 patients with T3 laryngeal carcinoma after treatment with transoral laser microsurgery. Head Neck. 2014 May;36(5):652-9.

11. Megwalu UC, Sikora AG. Survival outcomes in advanced laryngeal cancer. JAMA Otolaryngol Head Neck Surg. 2014 Sep;140(9):855-60.

12. Hartl DM, Ferlito A, Brasnu DF, Langendijk JA, Rinaldo A, Silver $\mathrm{CE}$, et al. Evidence-based review of treatment options for patients with glottic cancer. Head Neck. 2011 Nov;33(11):1638-48.

13. Edge S, Byrd DR, Compton CC, Fritz AG, Greene F, Trotti A. AJCC cancer staging handbook. 7th ed. New York: Springer; 2010.

14. Weller MD, Nankivell PC, McConkey C, Paleri V, Mehanna HM. The risk and interval to malignancy of patients with laryngeal dysplasia; a systematic review of case series and meta-analysis. Clin Otolaryngol. 2010 Oct;35(5):364-72.

15. El Dib R, Nascimento Junior P, Kapoor A. An alternative approach to deal with the absence of clinical trials: a proportional meta-analysis of case series studies. Acta Cir Bras. 2013 Dec;28(12):870-6.

16. Luo XN, Chen LS, Zhang SY, Lu ZM, Huang Y. Effectiveness of chemotherapy and radiotherapy for laryngeal preservation in advanced laryngeal cancer: a meta-analysis and systematic review. Radiol Med. 2015 Dec;120(12):1153-69.

17. Egger M, Davey Smith G, Schneider M, Minder C. Bias in meta-analysis detected by a simple, graphical test. BMJ. 1997 Sep;315(7109): 629-34.

18. Neyeloff JL, Fuchs SC, Moreira LB. Meta-analyses and forest plots using a Microsoft excel spreadsheet: step-by-step guide focusing on descriptive data analysis. BMC Res Notes. 2012 Jan;5:52.

19. Porter MJ, McIvor NP, Morton RP, Hindley AC. Audit in the management of T3 fixed-cord laryngeal cancer. Am J Otolaryngol. 1998 NovDec;19(6):360-4.

20. Kowalski LP, Batista MB, Santos CR, Scopel A, Salvajolli JV,Torloni H. Prognostic factors in T3,N0-1 glottic and transglottic carcinoma: a multifactorial study of 221 cases treated by surgery or radiotherapy. Arch Otolaryngol Head Neck Surg. 1996 Jan;122(1):77-82.

21. Foote RL, Olsen KD, Buskirk SJ, Stanley RJ, Suman VJ. Laryngectomy alone for T3 glottic cancer. Head Neck. 1994 Sep-Oct;16(5): 406-12.

22. Simpson D, Robertson AG, Lamont D. A comparison of radiotherapy and surgery as primary treatment in the management of T3 N0 M0 glottic tumours. J Laryngol Otol. 1993 Oct;107(10):912-5.

23. Woodhouse RJ, Quivey JM, Fu KK, Sien PS, Dedo HH, Phillips TL. Treatment of carcinoma of the vocal cord: a review of 20 years experience. Laryngoscope. 1981 Jul;91(7):1155-62.

24. Harwood AR, Hawkins NV, Beale FA, RiderWD, Bryce DP. Management of advanced glottic cancer: a 10 year review of the Toronto experience. Int J Radiat Oncol Biol Phys. 1979 Jun;5(6):899-904.

25. Nakayama M, Miyamoto S, Seino Y, Okamoto T, Kano K, Hasebe M, et al. One hundred supracricoid laryngectomies with cricohyoidoepiglottopexy: do we achieve better local control? Jpn J Clin Oncol. 2015 Aug;45(8):732-7.

26. Leszczynska M, Wierzbicka M, Tokarski M, Szyfter W. Attempt to improve functional outcomes in supracricoid laryngectomy in $\mathrm{T} 2 \mathrm{~b}$ and T3 glottic cancers. Eur Arch Otorhinolaryngol. 2015 Oct;272 (10):2925-31.

27. Day AT, Sinha P, Nussenbaum B, Kallogjeri D, Haughey BH. Management of primary T1-T4 glottic squamous cell carcinoma by transoral laser microsurgery. Laryngoscope. 2017 Mar;127(3):597-604.

28. Ansarin M, Cattaneo A, De Benedetto L, Zorzi S, Lombardi F, Alterio $\mathrm{D}$, et al. Retrospective analysis of factors influencing oncologic outcome in 590 patients with early-intermediate glottic cancer treated by transoral laser microsurgery. Head Neck. 2017 Jan;39(1): 71-81.
29. Peretti G, Piazza C, Penco S, Santori G, Del Bon F, Garofolo S, et al. Transoral laser microsurgery as primary treatment for selected T3 glottic and supraglottic cancers. Head Neck. 2016 Jul;38(7):1107-12.

30. Breda E, Catarino R, Monteiro E. Transoral laser microsurgery for laryngeal carcinoma: survival analysis in a hospital-based population. Head Neck. 2015 Aug;37(8):1181-6.

31. Pantazis D, Liapi G, Kostarelos D, Kyriazis G, Pantazis TL, Riga M. Glottic and supraglottic pT3 squamous cell carcinoma: outcomes with transoral laser microsurgery. Eur Arch Otorhinolaryngol. 2015 Aug;272(8):1983-90.

32. Vilaseca I, Bernal-Sprekelsen M, Luis Blanch J.Transoral laser microsurgery for T3 laryngeal tumors: prognostic factors. Head Neck. $2010 \mathrm{Jul} ; 32(7): 929-38$.

33. Motta G, Esposito E, Motta S, Tartaro G, Testa D. CO2 laser surgery in the treatment of glottic cancer. Head Neck. 2005 Aug;27(8):733.

34. Hinerman RW, Mendenhall WM, Morris CG, Amdur RJ, Werning JW, Villaret DB. T3 and T4 true vocal cord squamous carcinomas treated with external beam irradiation: a single institution's 35-year experience. Am J Clin Oncol. 2007 Apr;30(2):181-5.

35. Murakami R, Nishimura R, Baba Y, Yumoto E, Oya N, Yamashita Y. Concurrent chemoradiation therapy with low-dose CDDP and UFT for glottic carcinomas: evaluation using the sixth edition of the UICCTNM staging system. Acta Oncol. 2006;45(2):162-7.

36. Jorgensen K, Godballe C, Hansen O, Bastholt L. Cancer of the larynx: treatment results after primary radiotherapy with salvage surgery in a series of 1005 patients. Acta Oncol. 2002;41(1):69-76.

37. MacKenzie RG, Franssen E, Balogh JM, Gilbert RW, Birt D, Davidson J. Comparing treatment outcomes of radiotherapy and surgery in locally advanced carcinoma of the larynx: a comparison limited to patients eligible for surgery. Int J Radiat Oncol Biol Phys. 2000 Apr;47(1):65-71.

38. Mendenhall WM, Parsons JT, Mancuso AA, Pameijer FJ, Stringer SP, Cassisi NJ. Definitive radiotherapy for T3 squamous cell carcinoma of the glottic larynx. J Clin Oncol. 1997 Jun;15(6):2394-402.

39. Wang CC. Factors influencing the success of radiation therapy for T2 and T3 glottic carcinomas: importance of cord mobility and sex. Am J Clin Oncol. 1986 Dec;9(6):517-20.

40. Furusaka T, Matsuda A, Tanaka A, Matsuda H, Ikeda M. Superselective intra-arterial chemoradiation therapy for functional laryngeal preservation in advanced squamous cell carcinoma of the glottic larynx. Acta Otolaryngol. 2013 Jun;133(6):633-40.

41. Lin C, Sia E, Keller J. Concurrent chemoradiation for T3 glottic squamous cell carcinoma: a reasonable alternative to upfront laryngectomy.Adv Cancer Res Treat. 2012 Dec;2012:461217. https://doi. org/10.5171/2012.461217.

42. Chen AY, Fedewa S, Zhu J.Temporal trends in the treatment of earlyand advanced-stage laryngeal cancer in the United States, 1985-2007. Arch Otolaryngol Head Neck Surg. 2011 Oct;137(10):1017-24.

43. O’Neill CB, O’Neill JP, Atoria CL, Baxi SS, Henman MC, Ganly I, et al. Treatment complications and survival in advanced laryngeal cancer: a population-based analysis. Laryngoscope. 2014 Dec;124(12): 2707-13.

44. Laccourreye H, Laccourreye O, Weinstein G, Menard M, Brasnu D. Supracricoid laryngectomy with cricohyoidopexy: a partial laryngeal procedure for selected supraglottic and transglottic carcinomas. Laryngoscope. 1990 Jul;100(7):735-41.

45. Al-Gilani M, Skillington SA, Kallogjeri D, Haughey B, Piccirillo JF. Surgical vs nonsurgical treatment modalities forT3 glottic squamous cell carcinoma. JAMA Otolaryngol Head Neck Surg. 2016 Oct;142 (10):940-6.

46. Weinstein GS, El-Sawy MM, Ruiz C, Dooley P, Chalian A, El-Sayed $\mathrm{MM}$, et al. Laryngeal preservation with supracricoid partial laryngectomy results in improved quality of life when compared with total laryngectomy. Laryngoscope. 2001 Feb;111(2):191-9. 